www.jmscr.igmpublication.org

Impact Factor (SJIF): 6.379

Index Copernicus Value: 79.54

ISSN (e)-2347-176x ISSN (p) 2455-0450

crossrefDOI: https://dx.doi.org/10.18535/jmscr/v6i10.216

\title{
A Study on Dyslipidemia and the Atherogenic Index of Plasma in Chronic Kidney Disease
}

\begin{abstract}
Authors
Srividhya.S1, Sindhu', Anny Antony ${ }^{3}$

${ }^{1}$ Assistant Professor, Department of General Medicine, Kanyakumari Government Medical College,

Asaripallam

${ }^{2}$ Post Graduate, Department of General Medicine, Kanyakumari Government Medical College, Asaripallam

${ }^{3}$ Senior Resident, Department of General Medicine, Kanyakumari Government Medical College,

Asaripallam

Abstract

The purpose of the study is to study and analyse the atherogenicity of plasma in patients with Chronic Kidney Disease. This study was conducted on 85 patients of CKD admitted in Kanyakumari Government Medical College of age more than 18 years with renal function tests and ultrasound findings suggestive of $C K D$. The mean atherogenic index among CKD patients is .75 in our study. There is a significant change in the mean value of total cholestrol, TGL, and VLDL cholestrol among patients in various stages of CKD. A significant negative correlation was observed between eGFR and mean Atherogenic Index of Plasma.

Keywords: Atherogenic index of plasma (AIP), Chronic kidney disease-(CKD), Glomerular filtration rate - (GFR).
\end{abstract}

\section{Introduction}

Dyslipidemia is not uncommon among patients with chronic kidney disease. In chronic kidney disease patients, the common determinants of dyslipidemia could be $\mathrm{e}^{(1)}$

- Glomerular filtration rate (GFR)

- Co existence of diabetes mellitus

- Use of immune-suppressive agents

- Severity of proteinuria

- Type of treatment given(HD / PD / transplantation)

- Comorbidity

- Nutrition of the patient

In a chronic kidney disease patient, initial assessment of the serum lipid profile, primarily helps to confirm the diagnosis of elevations of the cholesterol and / or the triglyceride levels and also to determine the remediable secondary causes, if any. Major etiologies of secondary dyslipidemia $\operatorname{are}^{(1)}$

Medical Conditions

- Nephrotic syndrome

- Alcoholism

- Hypo-thyroidism

- Hepatic disease

- Diabetes

Medications

- 13-cis-retinoic acid

- Androgens 
- Antiepileptic drugs

- Oral contraceptive pills

- Highly active anti-retroviral therapy (HAART)

- Corticosteroids

- Cyclosporine

- Beta-antagonists

- Sirolimus

The crucial role of LDL-C as an indication for the initiation of pharmacological treatment using lipid lowering agents was emphasized in the previous guidelines. However, newer guidelines by the KDIGO Work Group ${ }^{(2)}$ advise against this.

\section{Materials and Methods}

- A minimum of 85 cases of CKD, who were admitted in Kanyakumari Government Medical College and Hospital between July and December 2017 were taken up for the study.

Chronic kidney disease was diagnosed based on history and clinical findings suggestive of CKD with supportive biochemical and sonological evidence is taken as criteria

\section{Inclusion criteria}

- Adults aged > 18 years with history and physical findings of kidney disease for a period above 6 months and

- Biochemistry suggestive of Chronic kidney disease.

- Ultrasound findings suggestive of Chronic kidney disease.

\section{Exclusion criteria}

- Patients already on lipid lowering agents.

- Patients on corticosteroids, androgens and oral contraceptive pills.

- Patients on HAART

- Patients on immune-suppressive drugs.

- Hypo-thyroid patients.

- Patients with coexistent liver disease

- Chronic alcoolics

- Patients with acute or chronic infection.

- Patients with nephrotic syndrome.

- Known Coronary Artery Diease patients

\section{Methodology}

Data Collection

- At the time of admission, general information of each patient (age, sex, weight, BMI, duration and nature of underlying renal disease, history of comorbid illness, treatment history if any, duration and frequency of haemodialysis (if applicable), personal history including smoking and alcoholism, familiar history of non -communicable disease and kidney disease) were recorded.

- Five $\mathrm{ml}$ of venous blood was taken aseptically from the ante-cubital fossa of the patient after fasting overnight for lipid profile determination.

- Other biochemical values like urea, creatinine and serum electrolytes along with the lipid parameters - total cholesterol, triglycerides, LDL and HDL cholesterol were also analysed.

- Estimated GFR was calculated using the Cockroft - Gault equation

$$
\mathrm{CrCl}=\frac{(140-\text { age })(\text { weight in } \mathrm{kg})}{(\text { Serum creatinine })(72)} \times 0.85 \text { if female }
$$

- Depending upon the glomerular filtration rate, chronic kidney disease patients were grouped into five stages

Stage $\mathrm{G} 1 \rightarrow$ GFR $\geq 90 \mathrm{ml} / \mathrm{min} / 1.73 \mathrm{~m}^{2}$

Stage G2 $\rightarrow$ GFR $60-89 \mathrm{ml} / \mathrm{min} / 1.73 \mathrm{~m}^{2}$

Stage G3a $\rightarrow$ GFR $45-59 \mathrm{ml} / \mathrm{min} / 1.73 \mathrm{~m}^{2}$

Stage G3b $\rightarrow$ GFR $30-44 \mathrm{ml} / \mathrm{min} / 1.73 \mathrm{~m}^{2}$

Stage G4 $\rightarrow$ GFR $15-29 \mathrm{ml} / \mathrm{min} / 1.73 \mathrm{~m}^{2}$

Stage G5 $\rightarrow$ GFR $<15 \mathrm{ml} / \mathrm{min} / 1.73 \mathrm{~m}^{2}$

- Blood samples for lipid profile estimation were ideally collected as a fasting blood sample. Fasting mainly affects the triglyceride values and to some extent the LDL - C values as estimated from the Friedwald formula. Fasting status does not affect the HDL $-\mathrm{C}$ levels ${ }^{(2)}$.

\section{Atherogenic index of plasma (AIP) ${ }^{(3)}$}

Atherogenic Index of Plasma (AIP) is a good predictor of the risk of atherosclerosis and 
ischemic heart disease ${ }^{(4)}$. AIP demonstrates the real relationship between the protective and the atherogenic lipoprotein and on the size of pre- and the anti- atherogenic lipoprotein particle ${ }^{(5)}$. AIP is calculated as logarithmic ratio of triglycerides to High Density Lipoprotein cholesterol log (TG/HDL-C)

$\begin{array}{lll}\text { Low risk of CVD } & \rightarrow & \text { AIP }<0.11 \\ \text { Intermediate risk } & \rightarrow & \text { AIP } 0.11-0.21 \\ \text { Increased risk } & \rightarrow & \text { AIP }>0.21^{(6)}\end{array}$

\section{Statistical Analysis}

The data collected was formulated into a master chart in Microsoft office Excel. Data analysis was performed using IBM-SPSS version 12.0 (IBMSPSS Science Inc., Chicago, IL).

Using this software, data are presented as mean, standard deviation, percentages, or the number of cases. Continuous data were compared by Independent student t-tests and One-way ANOVA and the correlation tested by Pearson correlation test. Significance was defined by $P$ values less than 0.05 using a two-tailed test.

\section{Observations and Results}

In our study, among the eighty five patients studied, fifty four patients were male and thirty one patients were female. Forty two among them were diabetics and thirty five were hypertensives. Thirty eight patients are treated with hemodialysis and the remaining forty seven patients are conservatively managed.

Fifteen patients had stage $3 \mathrm{CKD}$ with GFR between 30 and $59 \mathrm{ml} / \mathrm{min} / 1.73 \mathrm{~m}^{2}$. Thirty two patients had stage 4 disease with GFR between 15 and $29 \mathrm{ml} / \mathrm{min} / 1.73 \mathrm{~m}^{2}$. Thirty eight patients had stage $5 \mathrm{CKD}$ with GFR of less than 15 $\mathrm{ml} / \mathrm{min} / 1.73 \mathrm{~m}^{2}$

Table 1: Severity of CKD

\begin{tabular}{|lcc|}
\hline CKD stage & Frequency & Percent \\
\hline $\mathbf{3}$ & 15.00 & 17.65 \\
\hline $\mathbf{4}$ & 32.00 & 37.65 \\
\hline $\mathbf{5}$ & 38.00 & 44.70 \\
\hline Total & 85.00 & 100.00 \\
\hline
\end{tabular}

The lipid status in third fourth and fifth stages of chronic kidney disease is studied. The mean values of total cholesterol, triglyceride, VLDL - C and AIP varied significantly in the different stages of CKD, which showed a positive correlation.

Table 2: Stages of CKD

\begin{tabular}{|c|c|c|c|c|c|}
\hline & CKD stage & $\mathbf{N}$ & Mean & Std. Deviation & P Value \\
\hline \multirow[t]{3}{*}{ Total Cholesterol } & 3 & 15.00 & 193.27 & 16.58 & \multirow[t]{3}{*}{0.001} \\
\hline & 4 & 32.00 & 215.77 & 20.84 & \\
\hline & 5 & 38.00 & 221.55 & 26.51 & \\
\hline \multirow[t]{3}{*}{ TGL } & 3 & 15.00 & 192.93 & 47.09 & \multirow[t]{3}{*}{$<0.0001$} \\
\hline & 4 & 32.00 & 232.48 & 55.04 & \\
\hline & 5 & 38.00 & 270.18 & 46.51 & \\
\hline \multirow[t]{3}{*}{ LDL } & 3 & 15.00 & 95.60 & 6.57 & \multirow[t]{3}{*}{0.277} \\
\hline & 4 & 32.00 & 95.55 & 12.78 & \\
\hline & 5 & 38.00 & 106.13 & 25.14 & \\
\hline \multirow[t]{3}{*}{ HDL } & 3 & 15.00 & 43.07 & 5.74 & \multirow[t]{3}{*}{0.23} \\
\hline & 4 & 32.00 & 39.66 & 6.20 & \\
\hline & 5 & 38.00 & 40.66 & 6.62 & \\
\hline \multirow[t]{3}{*}{ VLDL } & 3 & 15.00 & 37.40 & 8.20 & \multirow[t]{3}{*}{$<0.0001$} \\
\hline & 4 & 32.00 & 44.61 & 9.92 & \\
\hline & 5 & 38.00 & 53.05 & 9.16 & \\
\hline \multirow[t]{3}{*}{ AIP } & 3 & 15.00 & 0.64 & 0.14 & \multirow[t]{3}{*}{$<0.0001$} \\
\hline & 4 & 32.00 & 0.76 & 0.13 & \\
\hline & 5 & 38.00 & 0.81 & 0.11 & \\
\hline
\end{tabular}




\section{JMSCR Vol||06||Issue||10||Page 1287-1292||October}

The below charts represent the same.

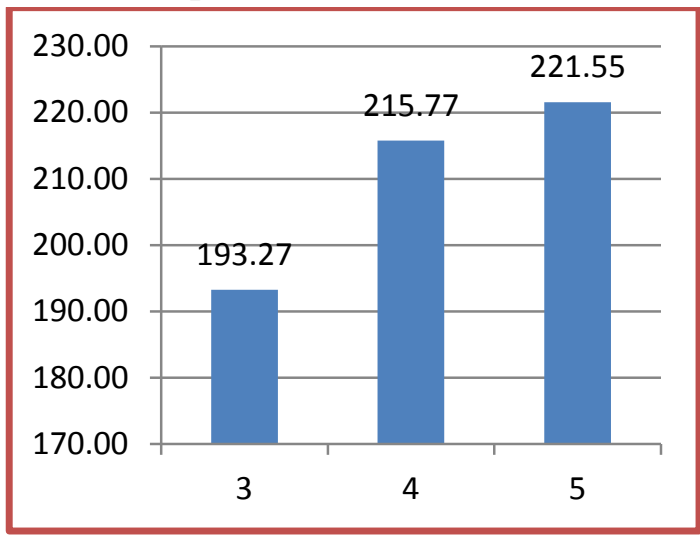

Fig 1: Total Cholesterol

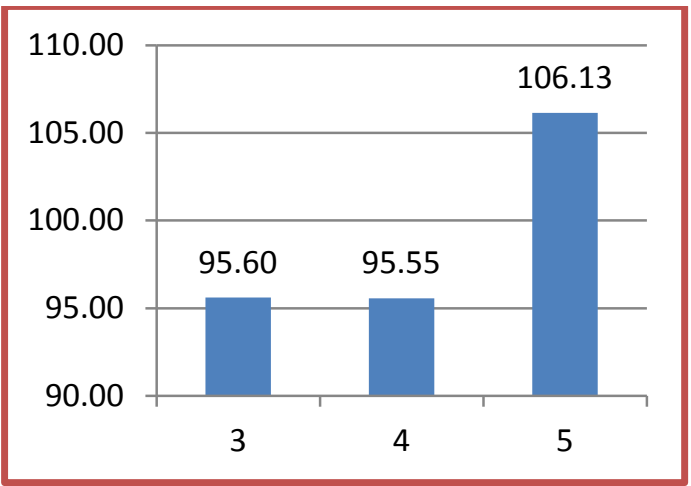

Fig 3: LDL

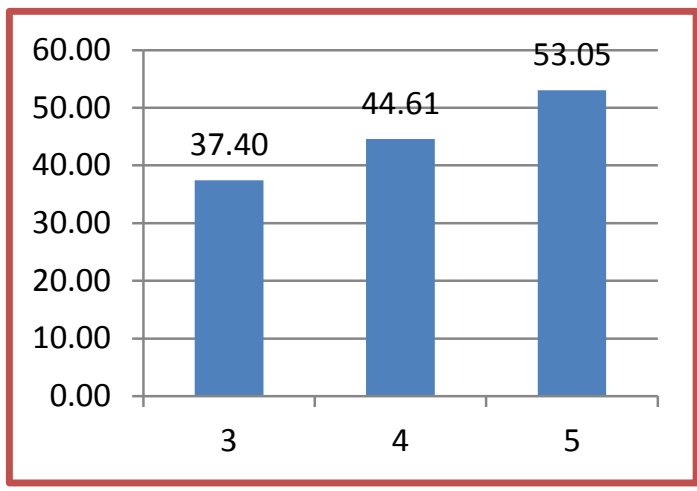

Fig 5: VLDL

The Pearson Correlation test was done to analyze the relationship between EGFR and AIP, which

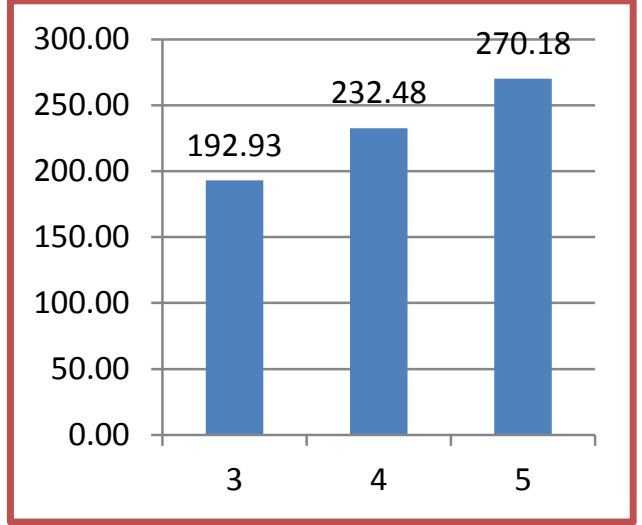

Fig 2: Triglycerides

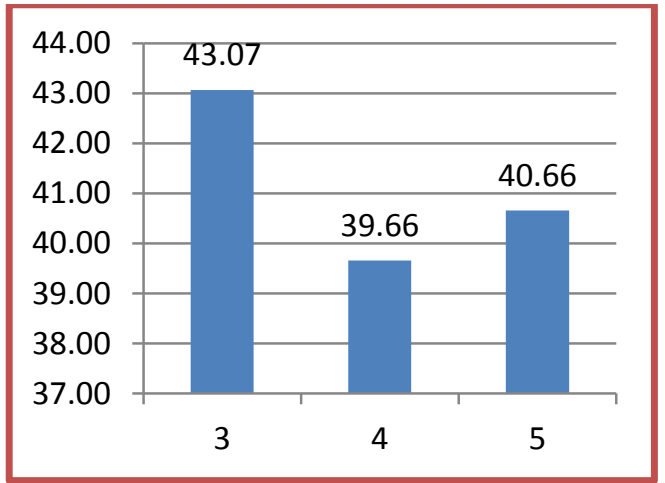

Fig 4: HDL

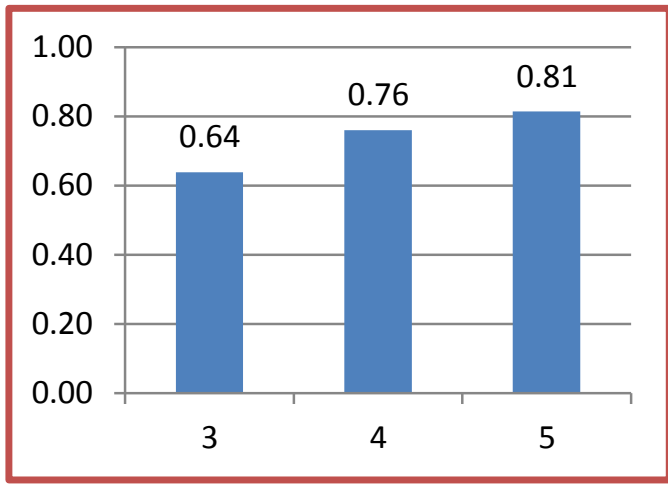

Fig 6: AIP

showed inverse correlation [with decreasing GFR values, there is an increase in the AIP values].

Table 3: EGFR Vs AIP

\begin{tabular}{|c|c|c|c|}
\hline & & Correlation coefficient & p-value \\
\hline EGFR & AIP & -0.506 & $<0.0001$ \\
\hline
\end{tabular}

\section{Discussion}

The leading etiology of hospital admissions and death in chronic kidney disease patients is cardiovascular disease. The spectrum of cardiovascular disease likely begins in the early stages of chronic kidney disease to reach critical level at the initiation of renal replacement therapy. Dyslipidemia, one of the traditional risk factors for coronary artery disease occurs frequently in chronic kidney disease ${ }^{(7)}$. 
However, the pattern of dyslipidemia in chronic kidney disease patients differs from the general population. Several studies were previously done which analyse the pattern of lipid levels in pre dialysis, dialysis and kidney transplanted patients. These type of studies help to improve knowledge about the lipid status in this high risk group and the subsequent cardiovascular risk.

Our study observes the pattern of dyslipidemia in ckd patients and finally the atherogenic index of the plasma [a value based on the triglyceride and HDL-C levels] is calculated and co-related with the stage of the kidney disease.

In our study population, the mean total cholesterol of the patients studied is $211.84 \mathrm{mg} / \mathrm{dl}$. The mean triglycerides is $241.47 \mathrm{mg} / \mathrm{dl}$. The mean LDL - C is $102.60 \mathrm{mg} / \mathrm{dl}$. The mean HDL $-\mathrm{C}$ is $42 \mathrm{mg} / \mathrm{dl}$. The mean VLDL - C is $47.08 \mathrm{mg} / \mathrm{dl}$. The mean atherogenic index of plasma is 0.75 .

The lipid status in stage 3,4 and 5 of kidney disease is studied. A significant variation is noted in the mean level of total cholesterol, triglyceride, VLDL - C and AIP in the various stages of CKD, which showed a positive correlation. However, no significant variation was noted in the LDL - C, HDL-C values.

The Pearson Correlation test was done to analyze the relationship between EGFR and AIP, which showed a significant negative correlation [with decreasing GFR values, there is an

In a similar study done by Rashmi Rekha et al, triglyceride and VLDL of the CKD group was elevated and the HDL - $\mathrm{C}$ was decreased when a comparison was made with the control group ${ }^{(8)}$.

Raju et al reported similar findings, which reported that triglyceride and VLDL of the CKD group was elevated and the HDL $-\mathrm{C}$ was decreased when compared with the control group. There is no significant variation in serum total cholesterol and Low Density Lipoprotein Cholesterol (LDL-C) among both the groups ${ }^{(9)}$.

Chijioke et al reported that TC, TG, HDL-C, LDL-C, and VLDL levels of the chronic kidney disease patients were markedly $(\mathrm{p}<0.05)$ different in both sexes on comparing with the control group. The cardiovascular risk indices such as TC/HDL-C, and LDL-C/HDL-C of the study group were more than that of the control group ${ }^{(10)}$. High triglyceride levels are probably due to marked elevations in the levels of apolipoprotein C-III, which in turn inhibits Lipoprotein lipase. LPL seen in the capillary endothelium, and causes the hydrolysis of VLDL and chylomicrons, and leads to deposition in arterial vessels. High levels of lipoprotein $\mathrm{Lp}(\mathrm{a})$ eventually accelerate atherosclerosis and cardiovascular disease in ckd patients. In CKD, plasma $\mathrm{Lp}$ (a) level is greatly affected by the glomerular filtration rate (GFR), and is elevated in the earlier stages of kidney disease. $^{(11)}$

CKD patients have a decreased function of lecithin cholesterol acyl-transferase (LCAT), which is linked to HDL. The action of LCAT is to convert cholesterol into its esters and to thus enable the hepatic removal of cholesterol. LCAT dysfunction leads to morphological changes in HDL, HDL assumes a more spherical form instead of its usual disc-shaped form, with resulting changes in its catabolism ${ }^{(11)}$.

Moreover, secondary hyperparathyroidism, which commonly occurs in CKD patients leads to downregulation of LP and LPL levels and worsens dyslipidemia $^{(12)}$

\section{Limitations of the Study}

1. The study was conducted in a small number of patients sand hence further studies with bigger sample size are needed.

2. The study lacked a non - CKD control group.

\section{Conclusion}

Lipid metabolism significantly varies in most patients with renal failure. Our results show that chronic kidney disease patients show significant abnormalities of lipid metabolism such as hypertriglyceridemia, hyper-cholesterolemia and low HDL cholesterol, which contribute to atherogenicity, and cardiovascular diseases, can increase the morbidity and mortality in CKD 
patients. As a first step of controlling dyslipidemia, bodyweight control, dietary regulation and regular exercise should be applied. Supplementing the diet with polyunsaturated fatty acids from fish oil to reduce triglycerides may be helpful. Statins can be used with careful monitoring. More extensive research on a larger population is required to confirm whether early evaluation and management could be helpful in the prevention of adverse clinical outcomes in CKD patients.

\section{Bibliography}

1. KDIGO. Lipid Work Group. KDIGO Clinical Practice Guideline for Lipid Management in chronic kidney disease. Kidney Inter., suppl. 2013; 3: 259 - 305.

2. KDIGO 2012. Clinical Practice Guidelines for the evaluation and management of chronic kidney disease. Volume - 3.Issue - 1. Jan 2013.

3. Shabnam Niroumand et al. Atherogenic Index of Plasma (AIP): A marker of cardiovascular Disease. Medical journal of Islamic Republic of Iran. July 2015.

4. Dobiášová $M$, Frohlich $J$, Šedová $M$, Cheung MC, Brown BG. Cholesterol esterification and atherogenic index of plasma correlate with lipoprotein size and findings on coronary angiography. J Lipid Res 2011;52(3):566-571. doi:10. 11.94/jlr.P011668

5. Dobiášová $M$, Frohlich J. The plasma parameter log (TG/HDL) as an atherogenic index: correlation with lipoprotein particle size and esterification rate in apoBlipoprotein-depleted plasma (FER HDL). Clinical Biochemistry 2001;34:583-588.

6. Dobiasova M. AIP - atherogenic index of plasma as a significant predictor of cardiovascular risk: from research to practice. VnitrLek 2006; 52(1):64-71

7. Balode et al. Serum lipid profile in chronic kidney disease patients on hemodialysis. Indian Journal of Applied Research.
Volume :3. Issue : 8. August 2013. (2022).

8. Rashmi Rekha et al. Unusual dyslipidemia in patients with chronic kidney diseases. Journal of clinical and diagnostic research. Jan 2017. Vol- 11(1). BC 01 - BC 04

9. Raju et al. A Study of lipid profile and lipid peroxidation in chronic kidney disease with special reference to hemodialysis. Journal of clinical research and bioethics.2013. 4:1

10. Chijioke et al. Lipid profile in chronic renal failure patients on dialysis. European Journal of Cardiovascular Medicine. Vol 2. Issue-11. 106-109.

11. Visconti et al. Lipid disorders in patients with renal failure : role in cardiovascular events and progression of chronic kidney disease. Journal of clinical and translational endocrinology. Vol 6. 2016. $8-14$.

12. Kasper et al. Harrison s Principles of Internal Medicine. $19^{\text {th }}$ edition. 